INPLASY

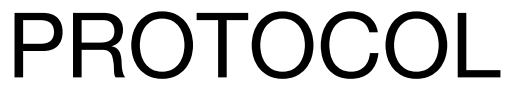

To cite: Wang et al. The efficacy and influencing factors of radioactive iodine in the treatment of patients with lung metastasis of differentiated thyroid cancer: a meta analysis. Inplasy protocol 2021110093. doi: 10.37766/inplasy2021.11.0093

Received: 24 November 2021

Published: 24 November 2021

Corresponding author: Jing Yu

13941156265@163.com

Author Affiliation:

Second Affiliated Hospital, Dalian Medical University, Dalian 116027, China.

Support: No.

Review Stage at time of this submission: Formal screening of search results against eligibility criteria.

Conflicts of interest:

None declared.

\section{The efficacy and influencing factors of radioactive iodine in the treatment of patients with lung metastasis of differentiated thyroid} cancer: a meta analysis

\author{
Wang, Y1; Jin, Z2; Zhang, F3; Mao, Y4.
}

Review question / Objective: To explore the efficacy and influencing factors of radioactive iodine in the treatment of patients with lung metastasis of differentiated thyroid cancer by meta-analysis.

Condition being studied: Lung is the most common distant metastasis site of differentiated thyroid cancer. Radioactive iodine therapy is the recommended method for the treatment of lung metastasis of differentiated thyroid cancer all over the world, but the influencing factors of its efficiency and efficacy are different in different studies. Therefore, we conducted a meta-analysis to explore the efficacy and influencing factors of radioactive iodine in the treatment of patients with lung metastasis of differentiated thyroid cancer.

INPLASY registration number: This protocol was registered with the International Platform of Registered Systematic Review and Meta-Analysis Protocols (INPLASY) on 24 November 2021 and was last updated on 24 November 2021 (registration number INPLASY2021110093).

\section{INTRODUCTION}

Review question / Objective: To explore the efficacy and influencing factors of radioactive iodine in the treatment of patients with lung metastasis of differentiated thyroid cancer by metaanalysis.

Condition being studied: Lung is the most common distant metastasis site of differentiated thyroid cancer. Radioactive iodine therapy is the recommended method for the treatment of lung metastasis of 
differentiated thyroid cancer all over the world, but the influencing factors of its efficiency and efficacy are different in different studies. Therefore, we conducted a meta-analysis to explore the efficacy and influencing factors of radioactive iodine in the treatment of patients with lung metastasis of differentiated thyroid cancer.

\section{METHODS}

Search strategy: Pubmed:(differentiated) AND ((( (Pulmonary Metastases[Title) Abstract]) OR (pulmonary metastasis[Title/ Abstract])) OR (lung metastasis[Title/ Abstract])) AND ((()(((radioactive iodine [ Title / A b stract]) OR (radioiodine[Title/Abstract])) OR (RAI[Title/ Abstract])) OR (131I[Title/Abstract])) OR (131-I[Title/Abstract])) OR (iodine[Title/ Abstract $]))$ AND $(()(()(()(()(()(()$ "Thyroid Neoplasms"[Mesh]) OR (Neoplasm, Thyroid[Title/Abstract])) OR (Thyroid Neoplasm[Title/Abstract])) OR (Neoplasms, Thyroid[Title/Abstract])) OR (Thyroid Carcinoma[Title/Abstract])) OR (Carcinoma, Thyroid[Title/Abstract])) OR (Carcinomas, Thyroid[Title/Abstract])) OR (Thyroid Carcinomas[Title/Abstract])) OR (Cancer of Thyroid[Title/Abstract])) OR (Thyroid Cancers[Title/Abstract])) OR (Thyroid Cancer[Title/Abstract])) OR (Cancer, Thyroid[Title/Abstract])) OR (Cancers, Thyroid[Title/Abstract])) OR (Cancer of the Thyroid[Title/Abstract])) OR (Cancer of the Thyroid[Title/Abstract])) OR (Adenoma, Thyroid[Title/Abstract])) OR (Adenomas, Thyroid[Title/Abstract])) OR (Thyroid Adenomas[Title/Abstract])))).

Participant or population: All over the world.

Intervention: Observational retrospective study, without intervention, was divided into effective group and ineffective group intervention.

Comparator: Observational retrospective study, without intervention, was divided into effective group and ineffective groupEffective or Ineffective.
Study designs to be included: Studies related to the efficacy and influencing factors of radioactive iodine in the treatment of differentiated thyroid cancer with lung metastasis were included

Eligibility criteria: (1) The subjects were patients with lung metastasis of differentiated thyroid cancer. (2) All patients had undergone thyroidectomy before 131I treatment. (3) The sample size is sufficient: $n>10$. (4) The study contains at least one influencing factor on the efficacy of 131I, and the extracted data can be made into a 2x2 four grid table.

Information sources: The following databases are systematically retrieved in the form of a combination of computer and manual: CNKI, Wanfang, VIP, Pubmed, Web of Science.

Main outcome(s): Total effective rate and Influencing factors: 1, Gender 2, Pulmonary nodule size 3, Pathological type 4, Cervical nodal metastases 5, Extrapulmonary distant metastases 6, CT or X-Ray result 7, lodine concentration within PM 8, Operation method 9, Extra thyroidal extension.

Quality assessment / Risk of bias analysis: Newcastle-Ottawa Scale (NOS) scale was used for quality evaluation.

Strategy of data synthesis: We will use Revman 5.4 which is recommended by Cochrane Collaboration Network for metaanalysis. The binary variable data are expressed by odds ratio (or) and 95\% confidence interval $(95 \% \mathrm{Cl})$. Cochran's Q test and I 2 test were used to test the heterogeneity among studies [9]. If $P>0.05$ or $12<50 \%$, it is considered that the heterogeneity among studies is not significant, and the fixed effect model (FEM) was used for combined analysis; If $P$ $<0.05$ and $I 2 \geq 50 \%$, it is considered that there is significant heterogeneity among studies, and the random effect model (REM) is used for combined analysis. Sensitivity analysis was performed to verify the stability of meta-analysis results. The 
overall efficacy results were expressed as risk difference (RD) and 95\% confidence interval (Cl).

Subgroup analysis: If the heterogeneity is significant and the source of heterogeneity cannot be found by sensitivity analysis, we will conduct subgroup analysis according to possible factors.

Sensitivity analysis: We will conduct sensitivity analysis on the total effective rate and each factor to verify the stability of the meta-analysis results. The specific method is to delete each study one by one and merge and analyze other studies.Sensitivity analysis was performed to verify the stability of meta-analysis results.

Language: Chinese or English.

Country(ies) involved: China.

Keywords: radioactive iodine, efficacy, lung metastasis, differentiated thyroid cancer, influencing factor influencing factors.

Contributions of each author:

Author 1 - Yizhen Wang.

Author 2 - Zhicheng Jin.

Author 3 - Fang Zhang.

Author 4 - Yangting Mao. 